\title{
Erratum to: Housing Policy and Building Types: From High Hopes to High-Rise
}

\author{
Amy Sargeant
}

\section{Erratum to: \\ P. Hirsch and C. O'Rourke (eds.), London on Film, Screening Spaces, https://doi.org/10.1007/978-3-319-64979-5_10}

The original version of this chapter was inadvertently published with the incorrect sub-heading "Grenfell Tower". This has been updated to "The Tower Block” (p. 137). 